\title{
3D false color computed tomography for diagnosis and follow-up of permanent denervated human muscles submitted to home-based Functional Electrical Stimulation
}

\author{
Ugo Carraro (1), Kyle J. Edmunds (2,3), Paolo Gargiulo (2,3) \\ (1) IRRCS Fondazione Ospedale San Camillo, Venezia, Italy; (2) Institute for Biomedical and \\ Neural Engineering, Reykjavik University, and (3) Landspítali, Reykjavík, Iceland
}

\begin{abstract}
This report outlines the use of a customized false-color 3D computed tomography (CT) protocol for the imaging of the rectus femoris of spinal cord injury (SCI) patients suffering from complete and permanent denervation, as characterized by complete Conus and Cauda Equina syndrome. This muscle imaging method elicits the progression of the syndrome from initial atrophy to eventual degeneration, as well as the extent to which patients' quadriceps could be recovered during four years of home-based functional electrical stimulation (h-b FES). Patients were pre-selected from several European hospitals and functionally tested by, and enrolled in the EU Commission Shared Cost Project RISE (Contract n. QLG5-CT-200102191) at the Department of Physical Medicine, Wilhelminenspital, Vienna, Austria. Denervated muscles were electrically stimulated using a custom-designed stimulator, large surface electrodes, and customized progressive stimulation settings. Spiral CT images and specialized computational tools were used to isolate the rectus femoris muscle and produce 3D and $2 \mathrm{D}$ reconstructions of the denervated muscles. The cross sections of the muscles were determined by 2D Color CT, while muscle volumes were reconstructed by 3D Color CT. Shape, volume, and density changes were measured over the entirety of each rectus femoris muscle. Changes in tissue composition within the muscle were visualized by associating different colors to specified Hounsfield unit (HU) values for fat, (yellow: [-200; -10]), loose connective tissue or atrophic muscle, (cyan: [-9; 40]), and normal muscle, fascia and tendons included, (red: $[41 ; 200])$. The results from this analysis are presented as the average HU values within the rectus femoris muscle reconstruction, as well as the percentage of these tissues with respect to the total muscle volume. Results from this study demonstrate that h-b FES induces a compliance-dependent recovery of muscle volume and size of muscle fibers, as evidenced by the gain and loss in muscle mass. These results highlight the particular utility of this modality in the quantitative longitudinal assessment of the responses of skeletal muscle to long-term denervation and h-b FES recovery.

Key Words: 3D Color Computed Tomography, False-color, permanent denervation diagnosis, human skeletal muscles, Functional Electrical Stimulation, Three-dimensional reconstruction, Modeling, Tissue composition
\end{abstract}

Eur J Transl Myol - Basic Appl Myol 2015; 25 (2): 129-140

The ever-expanding field of medical imaging utilizes a wide variety of techniques and processes to produce non-invasive images of various internal and external tissue morphologies. In the clinical context, medical imaging remains a vital tool for diagnostic and clinical investigations. Of the many facets of the field, most current research aims to improve aspects of instrumentation design, data acquisition methodology, image processing software, and computational modeling. Indeed, three-dimensional (3D) visualization of the internal anatomy provides valuable information for the diagnosis and surgical treatment of many pathologies, but every modality has its inherent limitations. ${ }^{1,2}$ For the purposes of clinical assessment in particular, visually simplistic imaging methods that can optimize the noninvasive, high-resolution assessment of diseased or damaged tissues have readily been identified as a strategic priority in clinical research, and extant imaging modalities have certainly been identified as preferential. $^{3-11}$ However, their 


\section{D Color CT of FES trained denervated human muscle}

Eur J Transl Myol - Basic Appl Myol 2015; 25 (2): 129-140

employment via standard methodology may not be optimal for various avenues of translational myology research. The implementation of traditional imaging modalities, in the context of a variety of novel case studies, can significantly impact this process of methodology optimization.

Here, we review the results of one such case study, involving the use of a customized false-color 3D computed tomography (CT) protocol for the imaging of the rectus femoris of spinal cord injury (SCI) patients suffering from complete and permanent denervation, as characterized by complete Conus and Cauda Equina syndrome. ${ }^{12-18}$ Home-based functional electrical stimulation (h-bFES) was applied in these persons using customized electrical stimulators with large electrodes that covered nearly the entirety of the thigh ventral surface. ${ }^{14-26}$ The use the reported falsecolor 3D CT imaging protocol highlights its utility in both confirming the diagnosis of long-term denervation and in quantifying the progression of muscle atrophy to degeneration. ${ }^{27-33}$ Furthermore, this method illuminates the importance of $\mathrm{h}-\mathrm{b}$ FES training compliance with respect to the rectus femoris recovery in such patients.

\section{Effects of long-lasting complete denervation of human muscles}

Long-standing, complete denervation of human muscles is most often the result of permanent lower motor neuron (LMN) death in patients with either acute damage, such as spinal cord injury (SCI), or pathological conditions, such as peripheral nerve lesions or neuromuscular disorders that result in severe muscular atrophy, apoptosis and fat degeneration of muscle tissue. ${ }^{34-42}$ In either case, the progression of denervation in muscle fibers begins with spontaneous activation, i.e. fibrillation, and leads to myofiber atrophy, ultrastructural changes of excitationcontraction coupling, and the gradual loss of excitability in response to external electrical stimulation using standard commercial stimulators. ${ }^{36}$ Finally, muscles enter severe atrophy, wherein myofibers undergo the internalization of subsarcolemmal myonuclei, resulting in the regrouping of tens of myonuclei within the center of myofibers and the complete disappearance of long segments of the contractile apparatus. ${ }^{13}$ Eventually, muscle fibers all but completely disappear, while fibrous and adipose tissue accumulates. ${ }^{14}$ Additionally, based on results of our world-unique CIR-Myo-Bank of LMN-denervated human muscle biopsies, the time course of human muscle atrophy and degeneration has been shown to occur over the order of years - not months, as was common belief based upon rodent models. ${ }^{42-44}$ Furthermore, both mid and late phase denervation present three very contrasting myofiber populations: beside those which are severely atrophic with internalized groups of myonuclei, there are large fasttype muscle fibers that continue to be present four-to- six years after SCI, ${ }^{45,46}$ and embryonic myosin heavy chains-positive myotubes and myofibers. The last events are evidence of muscle fibers regenerated through satellite cell activation, proliferation and fusion during the last month before muscle biopsy have been harvested, regardless of the time from SCI. ${ }^{1,47,48}$ $\mathrm{Up}$ to recent years, the LMN-denervated muscles of affected legs in paraplegic patients were not commonly treated with functional electrical stimulation (FES), because it was widely accepted that long-term and completely denervated muscles could not effectively respond to commercially available electrical stimulators due to the assumptive disappearance of a majority of muscle fibers in severely atrophic legs.

On the other hand, studies in animal models and humans indicate that denervation-induced severe atrophy and degeneration may occur several months or years later than generally believed. For example, in rats, severe atrophy was shown not to occur for at least four months. ${ }^{49-51}$ Likewise, in rabbits degeneration of muscle tissue did not appear during the first year of denervation. ${ }^{52-55}$ Indeed, our own recent findings show that rat muscle maintains L-type $\mathrm{Ca}^{2+}$ current and gene expression of the related proteins longer than functional contractile apparatuses. ${ }^{37}$ Additional investigations in human patients showed that irrecoverable muscle tissue degeneration can be delayed up to three years, and indeed light microscopy analysis showed that myofibers retain at least $30 \%$ of their initial size over this duration, confirming at least a two-year window for possible FES intervention. ${ }^{12-26}$ However, electron microscopy investigations on patients biopsies indicated that the decay of excitationcontraction contractile apparatus, i.e., of t-tubules and triads, starts within one year of denervation, suggesting that for the best results the h-b FES treatment must start as early as possible. ${ }^{14,15,17}$

\section{Investigating h-b FES treatment of LMN- denervated muscles}

Until recently, the mid-term progression of irreversible lower motor neuron denervation was comparatively much less studied that the long-term progression of muscle atrophy in upper motor neuron lesion patients. Hundreds of reports published in the literature describe studies of muscle properties up to three years following upper motor neuron spinal cord injury. Within the first month of injury, the thickness of the muscle bulk, measured by ultrasound, has been shown to decrease by up to $40 \%{ }^{56}$ However, after the first few months of significant muscle mass loss, studies show that the atrophy process slows down considerably. ${ }^{57-58}$ Indeed, a $50 \%$ stable atrophy in spastic incomplete paralysis is well documented up to two years after SCI. ${ }^{59-63}$ Information regarding patients up to 20 years after SCI was relatively unavailable before our published results showed stable muscle atrophy in long-term paraplegics with complete upper motor neuron lesion 3 to 20 years 


\section{D Color CT of FES trained denervated human muscle}

Eur J Transl Myol - Basic Appl Myol 2015; 25 (2): 129-140

after SCI. ${ }^{64}$. FES is also applied to complete thoracic SCI patients to activate their leg muscles under the SCI level. ${ }^{59-63}$ However, it should be noted that almost all of these studies were related to FES in thoracic-level paraplegics that responded with tetanic muscle contraction when stimulated with commercial electrical stimulators. ${ }^{65}$

All these features are in sharp contrast to the type of spinal cord injury that involves lower motor neurons lesion, because this causes actual denervation of the affected muscles. The disease process is particularly severe when a complete transverse SCI involves all the lower motor neurons of the affected muscles. This is precisely the case of complete, irreversible Conus and Cauda Equina syndrome, wherein patients exhibit complete LMN denervation of the leg muscles. ${ }^{66}$ Complete LMN denervated muscles are, indeed, soon unable to sustain tension during tetanic contractions induced by electrical stimulation, as the long-lasting severe atrophy of the permanently inactive muscles is worsened by the replacement of myofibers with adipocytes and collagen. ${ }^{13-18,67-69}$

As previously mentioned, there is a clear rationale for the existence of a window of opportunity to initiate muscle stimulation that may not only delay or avoid muscle degeneration in LMN-denervated patients, but could also recover contractility of the atrophic muscles. While several stimulators and rehabilitation strategies are commercially available for performing FES on patients with upper motor neuron damage due to SCI, for patients suffering from complete LMN damage, the protocols and home-based system developed and tested by the EU program "RISE" (Vienna, Austria) is the clear gold standard. ${ }^{17,18}$ This h-b FES system utilizes large surface electrodes fabricated from conductive polyurethane and custom-designed muscle stimulators to provide a homogeneous current distribution throughout the entire contact area. Two years of h-b FES training results in: 1) a significant increase of thigh muscle size and of the muscle fibers, with striking improvements of the ultra-structural organization of contractile structures and E-C coupling mechanisms; ${ }^{14-18}$ 2) a significant increase in muscle force output during electrical stimulation that is sufficient to perform h-b FES-assisted standing and inplace stepping exercises; ${ }^{14-18} 3$ ) evidence that the number and the size of recovered muscle fibers is inversely proportional to the elapsed time between a SCI event and the beginning of h-b FES. ${ }^{14-18}$ The reorganization of $\mathrm{T}$-tubules, $\mathrm{Ca}^{2+}$ release units, and myofibrils that follows h-b FES likely plays a role in the recovery of LMN denervated muscles, as well as their ability to be stimulated and to respond with tetanic contractions. $^{14-18}$

Patients were provided with stimulators and electrodes in order to perform stimulation at home for five days per week. The large $(180 \mathrm{~cm} 2)$ electrodes (Schuhfried $\mathrm{GmbH}$, Mödling, Austria), made of conductive polyurethane, were placed on the skin surface using a wet sponge cloth (early training) and fixed via elastic textile cuffs. As soon as the skin was accustomed to the necessary high current density, gel was used under the polyurethane electrodes to achieve minimal transition impedance. The electrodes were flexible enough to maintain evenly distributed pressure to the uneven and moving skin, thus providing homogeneous current distribution throughout the entire contact area. The particular h-b FES training strategy consisted of four combined stimulation programs. ${ }^{14-20}$ Additionally, since the progression of recovery by $h-b$ FES is inherently slow, patients were clinically evaluated every 12 weeks by physiatrists who progressively modified their training protocols according to the patient's improvements. ${ }^{16-18}$ It should likewise be noted that all applicable rules concerning the ethical use of human volunteers were followed during the course of this research (Approval of Ethical Committee, Vienna, Austria: EK-02-068-0702). For a multilingual translation of the work packages, ethical, and safety issues, visit: http://www.bio.unipd.it/bam/bam182\&3.html. ${ }^{25,26}$

\section{Muscle tissue segmentation and visualization using false-color 3D Computed Tomography}

Discrimination of tissue types, densities, and volumes is of particular interest in the medical imaging research community. The post-processing of 3D CT images via colorization of these morphologies holds particular utility with regards to the segmentation of skeletal muscle. Specific attenuation values are assigned to individual volumetric elements (voxels), based upon the degree to which CT X-rays of given energies transmit through the volume element. ${ }^{17,27-33}$ The degree of attenuation depends on the energy spectrum of the incident X-rays, as well as the average atomic number of the tissues of the patients. Since most computers utilize hardware that requires integer values, linear attenuation coefficients are rescaled to an integer range that encompasses 4096 values, between -1000 and +3095. This scale is known as the CT number, which may likewise be readily converted to Hounsfield units (HU). Comparatively dense tissues, such as bone, have large attenuation coefficients and thereby large, positive CT numbers; conversely, large, negative CT numbers are typical for low-density tissues, such as lung and adipose tissue. Muscles are normally displayed with CT number between 50 and 100 Hounsfield units, although within a normal muscle belly, other tissue elements are typically present, such as connective tissue and fat, which have much lower CT numbers. If a singular tissue type occupies a particular voxel, that element will possess a readilyidentifiable CT number corresponding singularly to that tissue type; however, typical voxels simultaneously contain several tissue types and thereby express an average CT number whose value is 


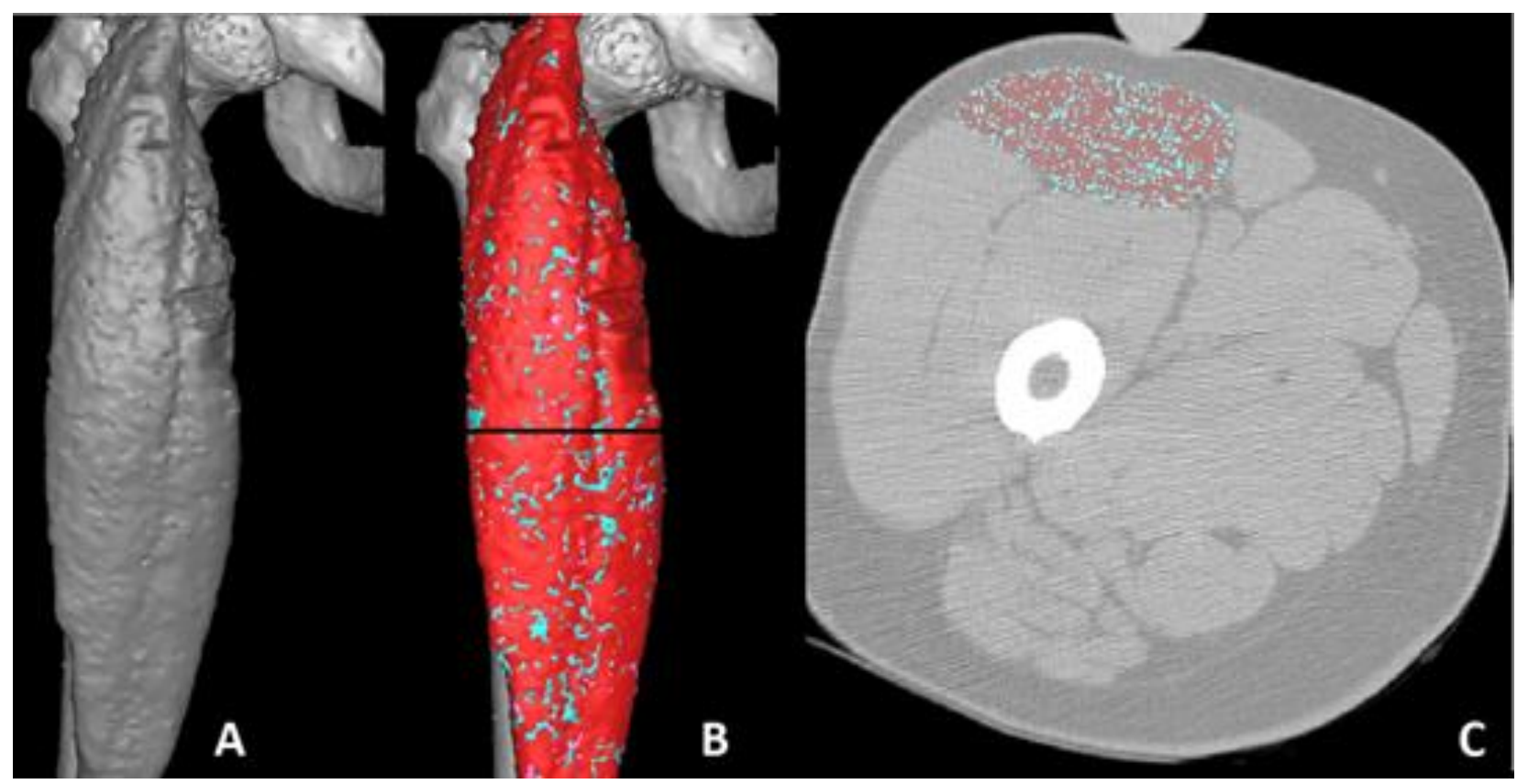

Fig 1. $3 D$ false-color CT reconstruction of a rectus femoris muscle from a healthy patient, including the epimysium at the cortical level in gray $(A)$ and false colors $(B)$, along with the corresponding cross-section at mid belly (C). The total volume is $4.2 \times 10^{5} \mathrm{~mm}^{3}$, and from this volume, $3 \%$ is fat [-200 to -10$] \mathrm{HU}, 28.7 \%$ is loose connective tissue and low-density (atrophied) muscle [-9 to 40] HU, and 68.3\% is normal muscle, if fascia and tendons are included [41 to 200] $\mathrm{HU}$.

proportional to the ratio of the tissue types. This phenomenon explains the wide range of values present within a particular dataset and suggests the necessity for increased voxel resolution and the development of novel intervoxel segmentation methods to optimize the study of muscle structural changes, in particular for 3D Color Muscle CT. ${ }^{27-33}$

\section{Protocol for 3D Color CT of long lasting muscle denervation}

Despite being widely used as an imaging modality in cardiology, ${ }^{1,2}$ the false color approach to spiral CT is typically ignored in clinical imaging-based evaluations of skeletal muscle tissue. As is typical of any spiral CT protocol, 3D data are gathered by scanning the patient's lower limbs with a spiral CT machine. For the purposes of this study, the imaging software MIMICS (http://www.materialise.com) was utilized to isolate and monitor the rectus femoris within the quadriceps muscle. 3D reconstruction is possible in the rectus femoris - even when severely degenerated due to longterm denervation, as the muscle remains readily recognizable despite severe atrophy of thigh muscles. As evidenced by Figure 1, a typical leg scan starts above the head of the femur and continues down to the knee joint, covering both legs with one scan. Slice increments are set to $0.625 \mathrm{~mm}$, resulting in a total of about 750-900 CT slices, depending on the patient's size. Each slice consists of $512 \times 512$ pixels, and each pixel has a gray value in the HU scale of 4096 grayscale values, meaning that pixels may be represented by a 12 bit value. This dataset is effectively a complete $3 \mathrm{D}$ description of the particular morphological structure, including all tissue types. The size of the volumetric element (voxel) in the dataset is about 0.7 $\mathrm{mm}$; therefore, as previously alluded to, the CT number assigned to these voxels often represented an average of different tissue elements. For instance, in the case of normal muscle tissue, such a voxel would contain the transverse section of 20 to 50 muscle fibers with their inter-myofiber elements (endomysium, nerve terminals, adipocytes, and, in particular, capillaries, arterioles, and venulae). It is important to note that the volume of a voxel corresponds approximately to one tenth of the volume accessed by a typical muscle needle biopsy, and that this slicing size allows for the microstructural evaluation of muscle quality via volume and density. The results of this microstructural analysis are presented as a 3D reconstruction containing the percentage of different tissues within the total volume of the rectus femoris muscle (Figure 1). In this reconstruction, only the first cortical layer of voxels describing the muscle epimysium is depicted. It should be stressed that these images depict the tissue distribution at the level of the muscle surface (epimysium), but do not coincide with the distribution through the entirety of the muscle volume. Therefore, to monitor and estimate the tissue composition of the 


\section{D Color CT of FES trained denervated human muscle}

Eur J Transl Myol - Basic Appl Myol 2015; 25 (2): 129-140

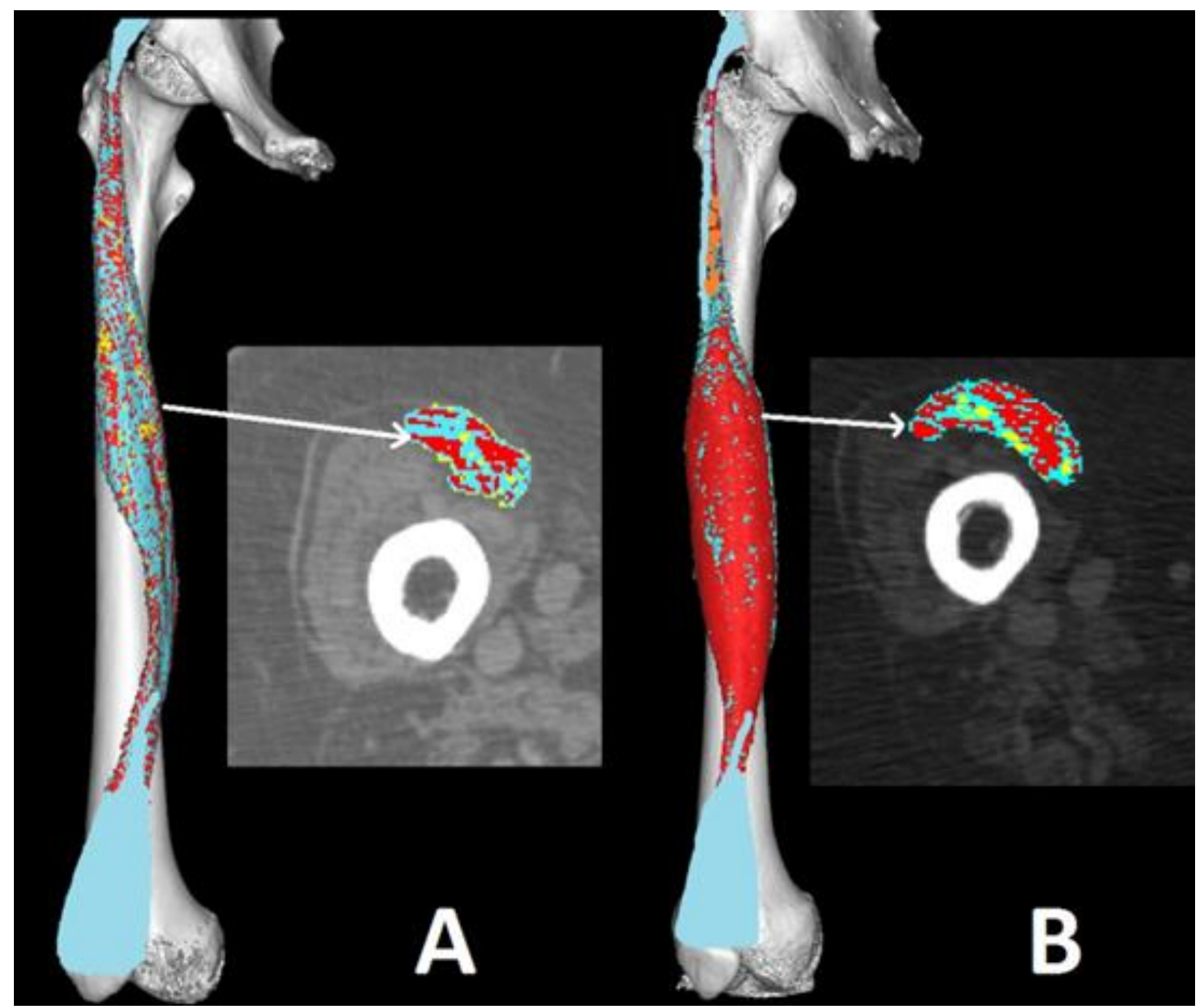

Fig 2. 3D reconstructions of rectus femoris from the representative SCI patient at the following three time points: A) four years after SCI, B) four years after starting $h$-b FES, and C) four years after discontinuing treatment. The topmost curve outlined as $a, b$, and c illustrates the increase and decrease of muscle volume at these time points, from $1.0 \times 10^{5} \mathrm{~mm}^{3}, 1.7 \times 10^{5} \mathrm{~mm}^{3}$, and $0.72 \times 10^{5} \mathrm{~mm}^{3}$, respectively. The primed curve below $(a$, $b^{\prime}$, and $\left.c^{\prime}\right)$ shows the increase and decrease of HU-based densities at these time points, from 42.5, 47.5, and 31.7, respectively. Note that the volumes are calculated identifying the epimysium, i.e. the cortical level of the muscle.

stimulated muscle volume, the computed tomography number present within the segmented volume were subdivided into three HU intervals and displayed with different colors as follows: [-200 to -10$]$, [-9 to 40], and [41 to 200] representing respectively: fat (yellow), loose connective tissue and atrophic muscle (cyan), and, if fascia and tendons are included, normal muscle (red).

\section{Evaluation of the impact of h-b FES}

This section details the results from the $3 \mathrm{D}$ segmentation and false-color analysis of both a healthy, active subject and a SCI patient with complete LMN denervation who was subjected to four years of h-b FES as previously described, but starting four years from SCI. Firstly, the mean HU value of normal leg muscle, collected from the healthy, young sportsman, was found to be between the mean density of the subpatellar tendon and the skin. The tendon density may, thus, be taken as the upper limit reference of the range of values for an healthy muscle tissue, as both exhibit a comparatively high protein content. Figure 2 illustrates the results from the false-color analysis of the SCI patient's rectus femoris after four years of denervation (Figure 2, panel A) and additional four years of h-b FES therapy, i.e., at eight years from SCI. Accordingly, Figure 2A shows that in a longitudinal 


\section{D Color CT of FES trained denervated human muscle}

Eur J Transl Myol - Basic Appl Myol 2015; 25 (2): 129-140
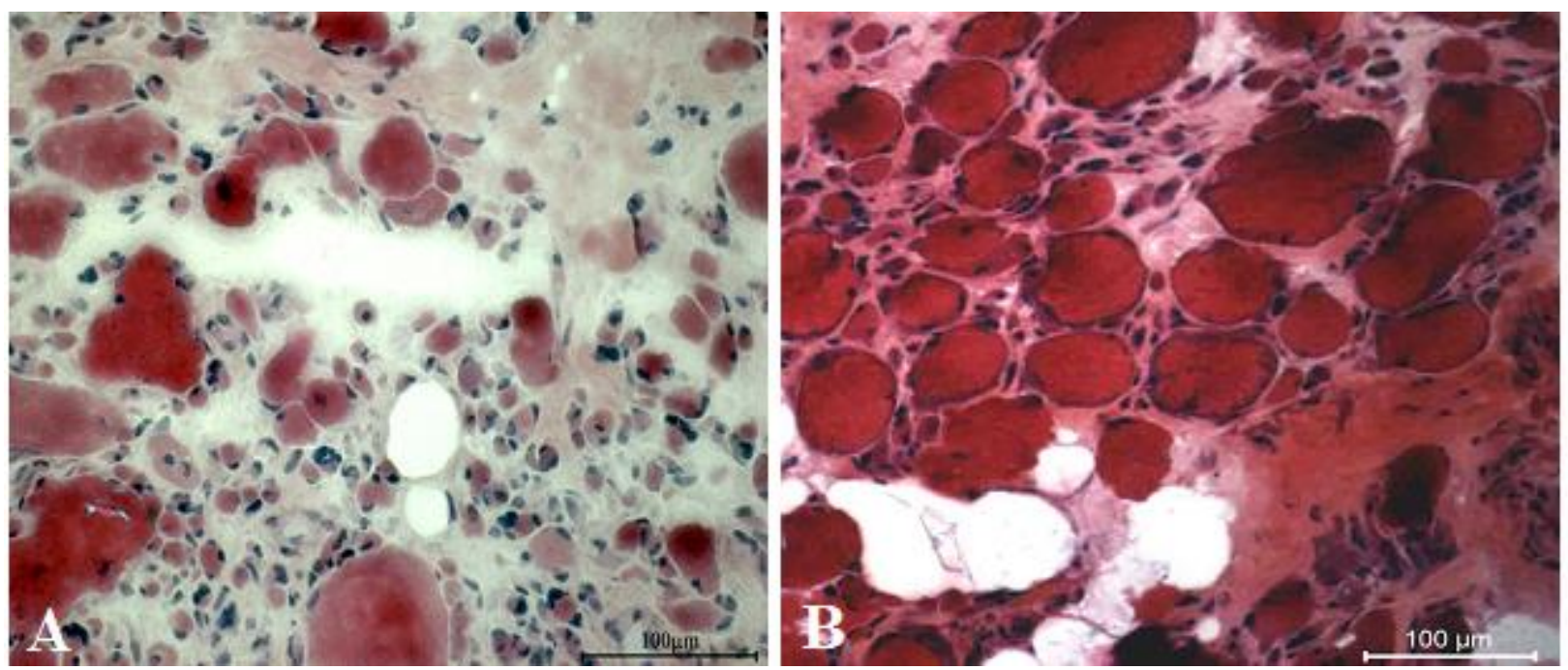

Fig 3. Histological analysis of the muscle biopsies harvested before (panel A) and after three years of h-b FES (panel B). Hematoxilin-Eosin stain. Interestingly, the area of the cryosections covered by muscle fibers profile in before and after $h-b$ FES specimens are $15 \%$ and $37 \%$, respectively, a behavior that is in good agreement with the results of the total volume of the rectus femoris before and after $h-b F E S$, respectively. Accordingly the sparse muscle fibers present before $h-b$ FES have mean diameter of less than $10 \mu m$, while the muscle diameter increase to $37 \mu \mathrm{m}$ in the after $h$-b FES biopsy. These results are in good agreement with the overall correlation tests among muscle strength, transverse CT area of the quadriceps muscle and diameter of the muscle fibers before and after $h-b$ FES in the series of LMN denervated person enrolled in the EU Project RISE. ${ }^{16,17}$ Bar $=100 \mu \mathrm{m}$.

study of our SCI patient, four years of denervation elicited a mean $\mathrm{HU}$ value of $42 \pm 20$, which is comparatively much less than that of the healthy sportsman (59 \pm 36$)$. Additionally, the volume of the rectus femoris was a full order of magnitude less than a typical healthy subject $\left(1.0 \times 10^{5} \mathrm{~mm}^{3}\right.$ for the SCI patient, compared to $4.2 \times 10^{5} \mathrm{~mm}^{3}$ computed previously in Figure 1). However, after four years of $h$ b FES, the SCI patient's muscle volume nearly doubled to $1.7 \times 10^{5} \mathrm{~mm}^{3}$. In addition, the three measured tissue compositions changed dramatically over the period of treatment: fat composition went from $8 \%$ to $4 \%$, loose connective tissue and atrophied muscle went from $47 \%$ to $36 \%$, and normal muscle went from $45 \%$ to $60 \%$. After four years of treatment, these muscular composition percentages were nearly recovered to those of the healthy adult example depicted in Figure 1 $(3 \%, 27.8 \%$, and $68.3 \%$ for fat, loose connective tissue and atrophied muscle, and normal muscle, respectively).

Figure 3 shows the results of the histological analysis of the muscle biopsies harvested before (panel A) and after three years of h-b FES (panel B). Interestingly, the area of the cryosections covered by muscle fibers profile in before and after h-b FES specimens are 15\% and $37 \%$, respectively a behavior that is in good agreement with the results of the total volume of the rectus femoris before and after h-b FES, respectively. Accordingly the sparse muscle fibers present before $\mathrm{h}$ - b FES have mean diameter of less than $10 \mu \mathrm{m}$, while the muscle diameter increase to $37 \mu \mathrm{m}$ in the after $\mathrm{h}-\mathrm{b}$ FES biopsy. These results are in good agreement with the overall correlation tests among muscle strength, transverse CT area of the quadriceps muscle and diameter of the muscle fibers before and after h-b FES in the series of LMN denervated person enrolled in the EU Project RISE. ${ }^{16,17}$ To better appreciate the extent of dependency of muscle recovery and its reversibility on compliance with h-b FES treatment, it may be useful to compare the h-b FES recovery of the four-yeardenervated muscle to its degeneration after four years of discontinued treatment (Figure 3). Intriguingly, the degree to which the atrophied rectus femoris recovered muscle volume was mirrored by the subsequent reversal of recovery after the discontinuation of $h-b$ FES (Figure 4).

As is evident in Figure 4, average HU values dropped from 47.5 to $31.7 \mathrm{HU}$ after discontinuation of h-b FES treatment. Likewise, muscle volume dropped from $1.7 \times 10^{5} \mathrm{~mm}^{3}$, and $0.72 \times 10^{5} \mathrm{~mm}^{3}$; both $\mathrm{HU}$ values and muscle volumes ended up substantially lower than they were prior to h-b FES treatment. The reported mean HU values are heavily influenced by the capillaries (open or closed) and content of fat (mean value -100 $\mathrm{HU}$ ), but also by the ratio of muscle sarcoplasm to extracellular fluid, which usually increases due to the absence of muscle contractions in the legs. Table 1 depicts the comparison between measured mean $\mathrm{HU}$ 


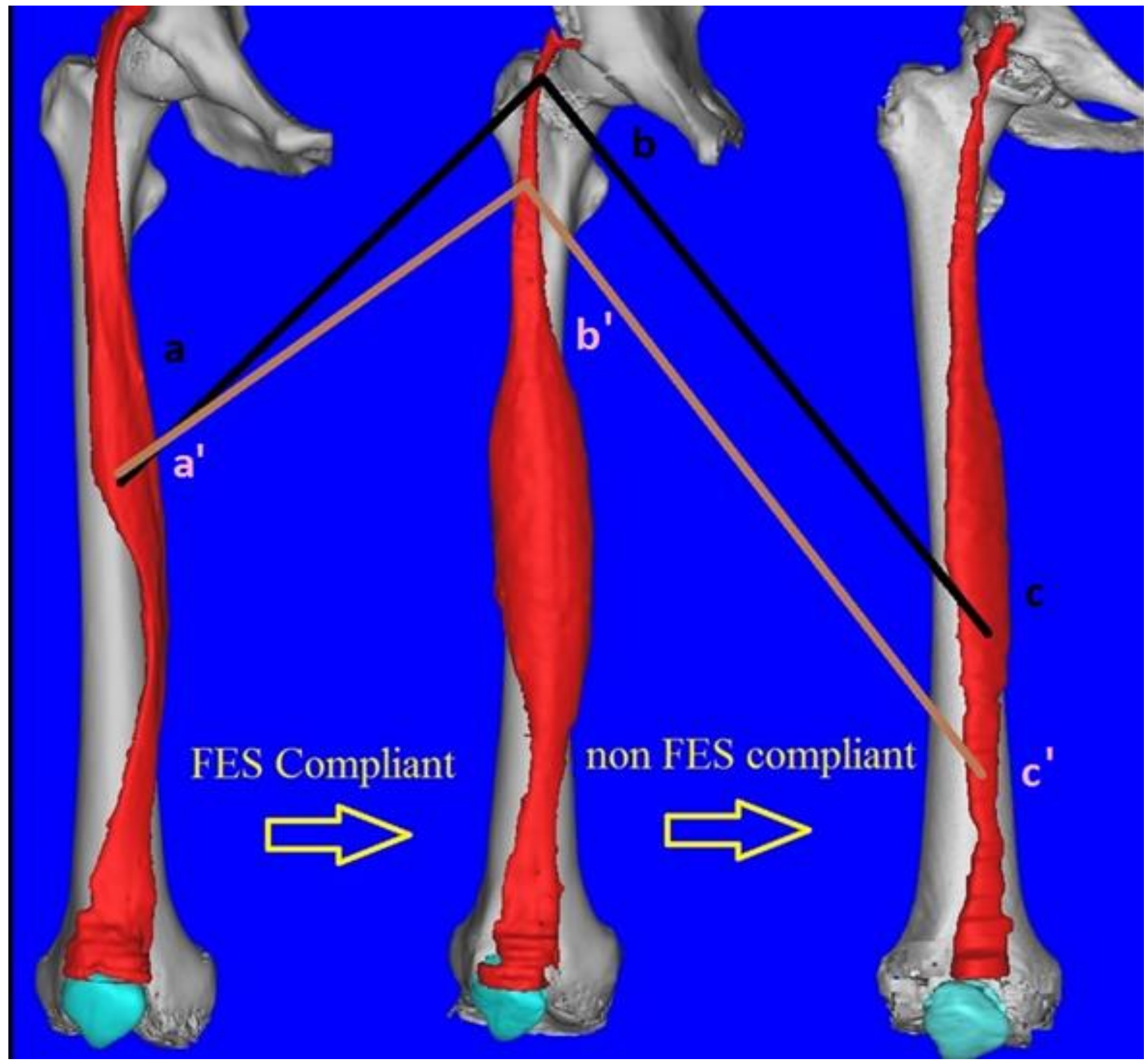

Fig 4. h-b FES-induced recovery of LMN denervated human muscles from atrophy/degeneration is fully dependent from patient's compliance.

$3 D$ reconstructions of rectus femoris from the SCI patient at three time points: $\boldsymbol{a}, \boldsymbol{a}$ ') four years after SCI, $\boldsymbol{b}, \boldsymbol{b}$ ') after four years of $h-b$ FES, and $\left.c, c^{\prime}\right)$ four years after discontinuing $h$ - $b$ FES training. The topmost curve outlined as $a, b$, and c illustrates the increase and decrease of muscle volume at these time points, from $1.0 \times 10^{5} \mathrm{~mm}^{3}$, $1.7 \times 10^{5} \mathrm{~mm}^{3}$, and $0,72 \times 10^{5} \mathrm{~mm}^{3}$, respectively. The primed curve below $\left(a^{\prime}, b^{\prime}\right.$, and $\left.c^{\prime}\right)$ shows the increase and decrease of HU-based densities at the same time points, from 42.5, 47.5, and 31.7, respectively. Note that the volumes are calculated identifying the epimysium, i.e. the cortical level of the muscle.

values of various tissues in the healthy, young sportsman and the SCI patient.

To begin discussing the utility of the h-b FES therapy, it must first be stressed that the amplitude of electrical current needed to extend the knee still remained more than 100-1000 times larger than that which is required for normally-innervated human muscles, demonstrating that the recovered function is not the results of reinnervation - a phenomenon which is not known to occur at such an extended time following SCI. ${ }^{63}$ In general, the case study highlights the utility of h-b FES for SCI patients with LMN denervation. Indeed, the muscle recovery in Figure 4 clearly illustrate an increase in rectus femoris volume and quality during four years of h-b FES training despite four years of prior LMN denervation. Likewise, after discontinuing 


\section{D Color CT of FES trained denervated human muscle}

Eur J Transl Myol - Basic Appl Myol 2015; 25 (2): 129-140

Table 1. Comparison of HU values from various tissues in both a healthy sportsman and a SCI patient.

\section{Tissues - Sportsman}

Subcutaneous fat

Skin

Muscle tissue

Sub-patellar tendon

Bone (femur)

\begin{tabular}{|c|c|c|}
\hline Mean HU & $+/-$ & SD \\
\hline-103 & & 34 \\
\hline 20 & & 100 \\
\hline 59 & & 36 \\
\hline 63 & & 39 \\
\hline 1014 & & 360 \\
\hline
\end{tabular}

\section{Tissues - SCI Patient}

Muscle tissue after 4 years from the start of denervation (SCI)

Muscle tissue after 4 years of h-b FES ( 8 years after SCI)

Muscle tissue after 4 years of detraining (12 years after SCI)

Note that each of the measurements were taken at the same location along the subject's femur.

therapy, our results clearly show worsening of rectus femoris degeneration, highlighting the importance of life-long sustaining h-b FES treatment in SCI patients. Important additional benefits for these patients include the improved cosmetic appearance of lower extremities, the enhanced cushioning effect for seating, and the overall reduction of leg edema. ${ }^{16}$ These benefits may additionally be extended to patients with similar lesions (incomplete LMN denervation of skeletal muscles) - especially with regards to determining whether h-b FES can reduce secondary complications related to disuse and impaired blood perfusion (reduction in bone density, risk of bone fracture, decubitus ulcers, and pulmonary thromboembolism). Hence, it is of special interest to monitor and study the electrophysiology of the rectus femoris - specifically with regards to the modeling and measurement of electrical stimulation effects. Indeed, our previous works in assessing segmentation techniques and related computational tools were used to isolate the rectus femoris from other proximal muscle bellies and analyze the muscle in a novel way. $^{27-33}$

In general, it may be recommended that h-b FES should be applied to all denervated leg muscles (from the gluteus to the foot muscles), but the main target muscle group for this therapy should be the quadriceps femoris, which consists of the four muscles on the front of the thigh: rectus femoris, vastus lateralis, vastus medialis and vastus intermedius. The muscle volume in the quadriceps is very large, and the muscles are not uniformly activated by external electrical stimulation. In fact, surface electrodes are by definition non-selective, and enormous amounts of energy is used to deliver electrical stimuli to denervated muscles. The rectus femoris occupies the middle of the thigh and covers most of the other three quadriceps muscles; hence, it is closer to surface electrodes and, therefore, more exposed to external stimulation - a notion which was evidenced in a study using T2 mapping. ${ }^{70,71}$

\section{Advantages and disadvantages of 3D Color CT imaging, segmentation, and reconstruction}

The particular computational method depicted herein is founded upon critical thresholding criteria and CT numbers, which are in-turn used to define different tissues within the muscle. The main advantage of using segmentation technique and $3 \mathrm{D}$ modeling is that it provides the option to analyze the whole muscle and not a small part of it. This notion is particularly valuable with respect to the presented case study, as we were able to readily isolate the rectus femoris as our muscle belly of interest. A further advantage of this method is that by adjusting the dimensions of the voxel size utilized, one may investigate various tissue compositions at the microscopic level. With this approach, the characteristics observed on the macroscopic level can be compared and correlated to measurements made on much smaller samples, and these results can serve as a validation of specific details from muscle biopsies. However, the main limitation for the use of this technique is related to the radiation dose, which the patient absorbs during the scanning process, and the overall reliability of CT numbers to discriminate tissue composition, as various physical factors can influence CT number representation during a scanning session. The parameter which mostly affects the accuracy and the spatial distribution of CT numbers is the applied voltage across an X-ray tube; this amplitude is measured in kilovolts and dictates the maximal X-ray energy and, therefore, the attenuation coefficient. CT number distributions are also influenced by phantom (or patient) orientation within the scan aperture. Therefore, it is necessary to recognize and account for these variabilities when CT numbers are employed for tissue characterization and comparison. Therefore, to avoid or at least limit possible discrepancies in CT number assignments, in the beginning of the clinical trial, a scanning protocol should be established and calibration tools employed 


\section{D Color CT of FES trained denervated human muscle}

Eur J Transl Myol - Basic Appl Myol 2015; 25 (2): 129-140

during every measurement (a rule of thumb that was followed in the presented study). Additionally, to limit the patient dose, spiral CT technology should be used to gather 3D data and volumes of interest in order to collect necessary information in the shortest possible period of time. We are confident that this case study, in accordance with other related works from the EU Program RISE, may help to convince clinicians to extend h-b FES observation to a larger cohort of cases. Specifically, the case study vividly highlights the use of 3D false-color $\mathrm{CT}$ as a non-invasive imaging procedure designed and implemented to objectively demonstrate the improvements in muscle mass and contractility, despite complete LMN denervation. The reported method describes a perspective opportunity for generating a multidisciplinary approach to monitor myopathies, based on this and other pre- and end-point analyses, such as muscle biopsy and functional muscle ultrasound analyses. ${ }^{72}$ All together, these methods may reopen the possibility for testing therapeutic rehabilitation approaches in shorter time frame studies. In conclusion, the study presented herein outlines the particular utility of this modality in quantitative longitudinal assessments of the responses of skeletal muscle to long-term denervation and to h-b FES induced recovery. Ongoing research is aimed to select other muscle views (multiple or mid-belly transverse section, multiple or mid-belly vertical section, etc.) to identify a panel of the most descriptive presentations. Additional analytical methods of muscle tissue volume and density may be found in other published clinical imaging articles, ${ }^{74-75}$ in studies of aging $^{76}$ and are described in detail in other reviews and articles of the EJTM Special "News on clinical imaging of muscle tissue", 77-81

\section{Acknowledgement}

This research has been supported by the University Hospital Landspitali Scientific Fund, the EU Commission Shared Cost Project RISE (Contract n. QLG5-CT-2001-02191), The Austrian Ministry of Science, and the Italian MIUR funds to the Laboratory of Translational Myology, University of Padova, Italy. U.C. thanks the Interdepartmental Research Center of Myology at the Department of Biomedical Sciences, University of Padova, Italy for collaboration and hospitality and the Ludwig Boltzmann Institute of Electrical Stimulation and Physical Rehabilitation of Vienna at the Department of Physical Medicine, Wilhelminenspital, Vienna, Austria for support and collaboration.

\section{Corresponding Author}

Dr. Paolo Gargiulo, Institute for Biomedical and Neural Engineering, Háskólinn í Reykjavík, Menntavegur 1 - 101 Reykjavík, Iceland

Email: paologar@landspitali.is
E-mails of Co-Authors:

Ugo Carraro, ugo.carraro@unipd.it

Kyle J. Edmunds, kylejedmunds@gmail.com

\section{References}

1. Agatston AS, Janowitz WR, Hildner FJ, et al. Quantification of coronary artery calcium using ultrafast computed tomography. J Am Coll Cardiol 1990;15:827-32. doi:10.1016/0735-1097 (90)90282-T

2. Ameli-Renani S, Rahman F, Nair A, et al. Dualenergy CT for imaging of pulmonary hypertension: challenges and opportunities. Radiographics 2014;34:1769-90. doi: 10.1148/ rg.347130085.

3. Stringari C, Cinquin A, Cinquin O, et al. Phasor approach to fluorescence lifetime microscopy distinguishes different metabolic states of germ cells in a live tissue. Proc Natl Acad Sci U S . 2011;108:13582-7.

4. Kasischke KA, Vishwasrao HD, Fisher PJ, et al. Neural activity triggers neuronal oxidative metabolism followed by astrocytic glycolysis. Science 2004;305(5680):99-103.

5. Skala MC, Riching KM, Gendron-Fitzpatrick A, et al. In vivo multiphoton microscopy of NADH and FAD redox states, fluorescence lifetimes, and cellular morphology in pre-cancerous epithelia. Proc Natl Acad Sci USA 2007;104:19494-9. Epub 2007 Nov 27.

6. König K, Uchugonova A, Gorjup E. Multiphoton fluorescence lifetime imaging of 3D-stem cell spheroids during differentiation. Microsc Res Tech 2011;74:9-17.

7. Bird DK, Vrotsos KM, Eliceiri KW, et al. Metabolic mapping of MCF10A human breast cells via multiphoton fluorescence lifetime imaging of the coenzyme NADH. Cancer Res 2005;65:8766-73.

8. Uchugonova A, König K. Two-photon autofluorescence and second-harmonic imaging of adult stem cells. J Biomed Opt 2008;13:054068. doi: $10.1117 / 1.3002370$.

9. Guo HW, Chen CT, Wei YH, et al. Reduced nicotinamide adenine dinucleotide fluorescence lifetime separates human mesenchymal stem cells from differentiated progenies. $\mathrm{J}$ Biomed Opt 2008;13:050505. doi: 10.1117/1.2990752.

10. Rice WL, Kaplan DL, Georgakoudi I. Twophoton microscopy for non-invasive, quantitative monitoring of stem cell differentiation. PLoS ONE 2010;5:e10075. doi: 10.1371/journal. pone. 0010075 .

11. Kantelhardt SR, Leppert J, Krajewski J, et al. Imaging of brain and brain tumor specimens by time-resolved multiphoton excitation microscopy ex vivo. Neuro-oncol 2007;9:103-12. Epub 2007 Feb 26. 


\section{D Color CT of FES trained denervated human muscle}

Eur J Transl Myol - Basic Appl Myol 2015; 25 (2): 129-140

12. Rossini K, Zanin ME, Carraro U. To stage and quantify regenerative myogenesis in human longterm permanent denervated muscle. Basic Appl Myol 2002; 12:277-86.

13. Kern H, Carraro U, Biral D, et al. Severely atrophic muscle fibers with nuclear clumps survive many years in permanently denervated human muscle. The Open Pathology Journal 2009;3:106-10.

14. Kern H, Boncompagni S, Rossini K, et al. Longterm denervation in humans causes degeneration of both contractile and excitation-contraction coupling apparatus that can be reversed by functional electrical stimulation (FES). A role for myofiber regeneration? J Neuropath Exp Neurol 2004;63:919-31.

15. Boncompagni S, Kern $\mathrm{H}$, Rossini $\mathrm{K}$, et al. Structural differentiation of skeletal muscle fibers in the absence of innervation in humans. Proc Natl Acad Sci U S A 2007;104:19339-44.

16. Kern H, Carraro U, Adami N, et al. One year of home-based Functional Electrical Stimulation (FES) in complete lower motor neuron paraplegia: Recovery of tetanic contractility drives the structural improvements of denervated muscle. Neurol Res 2010;32:5-12.

17. Kern H, Carraro U, Adami N, et al. Home-based Functional Electrical Stimulation (h-bFES) recovers permanently denervated muscles in paraplegic patients with complete lower motor neuron lesion. Neurorehab Neur Rep 2010;24:709-21.

18. Kern H, Carraro U. Home-based Functional Electrical Stimulation (h-b FES) for long-term denervated human muscle: History, basics, results and perspectives of the Vienna Rehabilitation Strategy. Eur J Transl Myol - Basic Appl Myol 2014;24:27-40.

19. Kern H, Hofer C, Strohhofer M, et al. Standing up with denervated muscles in humans using functional electrical stimulation. Artif Organs 1999;23:447-52.

20. Mayr W, Bijak M, Rafolt D, et al. Basic design and construction of the Vienna FES implants: Existing solutions and prospects for new generations of implants. Med Eng Phys 2001;23:53-60.

21. Kern H, Hofer C, Modlin M, et al. Denervated muscles in humans: Limitations and problems of currently used functional electrical stimulation training protocols. Artif Organs 2002;26:216-8.

22. Hofer C, Mayr W, Stöhr H, et al. A stimulator for functional activation of denervated muscles. Artif Organs 2002;26:276-9.

23. Kern H, Rossini K, Carraro U, et al. Muscle biopsies show that FES of denervated muscles reverses human muscle degeneration from permanent spinal motoneuron lesion. J Rehabil Res Dev 2005;42:43-3.

24. Kern H, Carraro U, Eds. Translational Myology Focus on Clinical Challenges of Functional Electrical Stimulation of Denervated Muscle. Basic Appl Myol 2008;18:38-100.

25. Kern H, Hofer C, Mayr W. Protocols for Clinical Work Package of the European Project RISE. Basic Appl Myol/Eur J Transl Myol 2008;18:3944.

26. Kern H, Hofer C, Mayr W, et al. European Project RISE: Partners, protocols, demography. Basic Appl Myol/ Eur J Transl Myol 2009;19:211-6.

27. Helgason T, Gargiulo P, Johannesdotir F, et al. Monitoring muscle growth and tissue changes induced by electrical stimulation of denervated degenerated muscles with CT and stereolithographic 3D modeling. Artif Organs 2005;29:440-3

28. Gargiulo $\mathrm{P}$, Helgason $\mathrm{T}$, Ingvarsson $\mathrm{P}$, et al. Morphological changes in denervated muscle treated with FES. Basic Appl Myol 2007;17:1336.

29. Gargiulo P, Vatnsdal B, Ingvarsson P, et al. Restoration of muscle volume and shape induced by electrical stimulation of denervated degenerated muscles: qualitative and quantitative measurement of changes in rectus femoris using computer tomography and image segmentation. Artif Organs 2008;32:609-13.

30. Gargiulo P. 3D modelling and monitoring of denervated muscle under functional electrical stimulation treatment and associated bone structural changes. Doctoral thesis, Vienna University of Technology, Vienna, Austria, 2008.

31. Gargiulo $\mathrm{P}$, Vatnsdal $\mathrm{B}$, Ingvarsson $\mathrm{P}$, et al. Computational methods to analyse tissue composition and structural changes in denervated muscle undergoing therapeutic electrical stimulation. Basic Appl Myol/ Eur J Transl Myol 2009;19:157-62.

32. Gargiulo P, Kern H, Carraro U, et al. Quantitative colour 3D CT imaging of human long-term denervated muscle. Progression to fibrosis of perimysium and a case report of FES recovery. Neurol Res 2010;32:13-9.

33. Gargiulo P. 3D Modelling and monitoring of denervated muscle under Functional Electrical Stimulation treatment and associated bone structural change. Eur J Transl Myol - Basic Appl Myol 2011;21:31-94.

34. Morawietz C, Moffat F. Effects of locomotor training after incomplete spinal cord injury: a systematic review. Arch Phys Med Rehabil. 2013;94:2297-308. doi: 10.1016/j.apmr.2013.06. 23. Epub 2013 Jul 9. 


\section{D Color CT of FES trained denervated human muscle}

Eur J Transl Myol - Basic Appl Myol 2015; 25 (2): 129-140

35. Adami N, Kern H, Mayr W, et al. Permanent denervation of rat Tibialis Anterior after bilateral sciatectomy: determination of chronaxie by surface electrode stimulation during progression of atrophy up to one year. Basic Appl Myol 2007; 17:237-43.

36. Abruzzo PM, Di Tullio S, Marchionni C, et al. Oxidative stress in the denervated muscle. Free Radic Res 2010;44:563-76. doi: 10.3109/ 10715761003692487.

37. Squecco R, Carraro U, Kern H, et al. Despite lost contractility, a sub-population of rat muscle fibers maintains an assessable excitation-contraction coupling mechanism after long-standing denervation. J Neuropath Exp Neurol 2009;68:1256-68.

38. Carraro U, Franceschi C. Apoptosis of skeletal and cardiac muscles and physical exercise. Aging (Milano) 1997;9:19-34. Review.

39. Sandri M, Carraro U. Apoptosis of skeletal muscles during development and disease. Int $\mathbf{J}$ Biochem Cell Biol 1999;31:1373-90.

40. Dirks A, Leeuwenburgh C. Apoptosis in skeletal muscle with aging. Am J Physiol Regul Integr Comp Physiol 2002;282:R519-27.

41. Piepoli MF, Guazzi M, Boriani G, et al. Working Group 'Exercise Physiology, Sport Cardiology and Cardiac Rehabilitation', Italian Society of Cardiology. Exercise intolerance in chronic heart failure: mechanisms and therapies. Part I. Eur J Cardiovasc Prev Rehabil 2010;17:637-42.

42. Carlson BM. The Denervated Muscle: 45 years later. Neurol Res 2008;30:119-122.

43. Carlson BM. The biology of long-term denervated skeletal muscle. Eur J Transl Myol Basic Appl Myol 2014;24:5-12.

44. Lomo T. The response of denervated muscle to long-term stimulation (1985, revisited here in 2014). Eur J Transl Myol - Basic Appl Myol 2014;24:13-9.

45. Biral D, Kern H, Adami N, et al. Atrophyresistant fibers in permanent peripheral denervation of human skeletal muscle. Neurol Res 2008;30:137-44.

46. Squecco R, Carraro U, Kern H, et al. Despite lost contractility, a sub-population of rat muscle fibers maintains an assessable excitation-contraction coupling mechanism after long-standing denervation. J Neuropath Exp Neurol 2009;68:1256-68.

47. Borisov AB, Dedkov EI, Carlson BM. Abortive myogenesis in denervated skeletal muscle: differentiative properties of satellite cells, their migration, and block of terminal differentiation. Anat Embryol 2005;209:269-279.

48. Carraro U, Rossini K, Mayr W, et al. Muscle fiber regeneration in human permanent lower motoneuron denervation: relevance to safety and effectiveness of FES-training, which induces muscle recovery in SCI subjects. Artif Organs 2005;29:87-91.

49. Carraro U, Boncompagni S, Gobbo V, et al. Persistent muscle fiber regeneration in long term denervation. Past, present, future. Eur J Transl Myol - Basic Appl Myol 2015;25:77-92.

50. Schmalbruch H, al-Amood WS, Lewis DM. Morphology of long-term denervated rat soleus muscle and the effect of chronic electrical stimulation. J Physiol (Lond) 1991;441:233-241.

51. Carraro U, Morale D, Mussini I, et al. Chronic denervation of rat diaphragm: maintenance of fiber heterogeneity with associated increasing uniformity of myosin isoforms. J Cell Biol 1985;100:161-74.

52. Ashley Z, Sutherland $\mathrm{H}$, Lanmüller $\mathrm{H}$, et al. Determination of the chronaxie and rheobase of denervated limb muscles in conscious rabbits. Artif Organs 2005;29:212-5.

53. Ashley Z, Sutherland $\mathrm{H}$, Lanmuller $\mathrm{H}$, et al. Atrophy, but not necrosis, in rabbit skeletal muscle denervated for periods up to one year. Am J Physiol Cell Physiol 2007;292:C440-51.

54. Ashley Z, Salmons S, Boncompagni S, et al. Effects of chronic electrical stimulation on longterm denervated muscles of the rabbit hind limb. J Muscle Res Cell Motil 2007;28:203-17.

55. Ashley Z, Sutherland H, Russold MF, et al. Therapeutic stimulation of denervated muscles: the influence of pattern. Muscle Nerve 2008;38:875-86.

56. Gorgey SA, Dudley GA. Skeletal muscle atrophy and increased intramuscular fat after incomplete spinal cord injury. Spinal Cord 2007;45:304-9.

57. Lotta S, Scelsi R, Alfonsi E, et al. Morphometric and neurophysiological analysis of skeletal muscle in paraplegic patients with traumatic cord lesion. Paraplegia 1991;29:247-52.

58. Taylor PN, Ewins DJ, Fox B, et al. Limb blood flow, cardiac output and quadriceps muscle bulk following spinal cord injury and the effect of training for the Odstock functional electrical stimulation standing system. Paraplegia 1993;3:303-10.

59. Andersen JL, Mohr T, Biering-Sørensen F, et al. Myosin heavy chain isoform transformation in single fibers from $\mathrm{m}$. vastus lateralis in spinal cord injured individuals: effects of long-term functional electrical stimulation (FES). Pflugers Arch 1996;431:513-8.

60. Adams MM, Ditor DS, Tarnopolsky MA, et al. The effect of body weight-supported treadmill training on muscle morphology in an individual with chronic, motor-complete spinal cord injury: A case study. J Spinal Cord Med 2006;29:167-71.

61. Castro MJ, Apple DF, Jr, Staron RS, et al. Influence of complete spinal cord injury on 


\section{D Color CT of FES trained denervated human muscle}

Eur J Transl Myol - Basic Appl Myol 2015; 25 (2): 129-140

skeletal muscle within 6 mo of injury. J Appl Physiol 1999;86:350-8.

62. Crameri RM, Weston AR, Rutkowski S, et al. Effects of electrical stimulation leg training during the acute phase of spinal cord injury: A pilot study. Eur J Appl Physiol 2000;83:409-15.

63. Giangregorio LM, Webber CE, Phillips SM, et al. Can body weight supported treadmill training increase bone mass and reverse muscle atrophy in individuals with chronic incomplete spinal cord injury? Appl Physiol Nutr Metab 2006;31:283291.

64. Kern H, Hofer C, Modlin M, et al. Stable muscle atrophy in long- term paraplegics with complete upper motor neuron lesion from 3- to 20-year SCI. Spinal Cord 2008;46:293-304.

65. Mohr T, Andersen JL, Biering-Sorensen F, et al. Long-term adaptation to electrically induced cycle training in severe spinal cord injured individuals. Spinal Cord 1997;35:1-16.

66. Harrop JS, Hunt GE Jr , Vaccaro AR. Cauda Equina Syndrome. Conus medullaris and cauda equina syndrome as a result of traumatic injuries: management principles. Neurosurgical Focus 2004;16:1-23.

67. Kern H, Salmons S, Mayr W, et al. Recovery of long-term denervated human muscles induced by electrical stimulation. Muscle Nerve 2005; 31: 98-101.

68. Modlin M, Forstner C, Hofer C, et al. Electrical stimulation of denervated muscles: first results of a clinical study. Artif Organs 2005;29:203-6.

69. Dulhunty AF, Gage PW. Excitation-contraction coupling and charge movement in denervated rat extensor digitorum longus and soleus muscle. $\mathbf{J}$ Physiol 1985;358:375-89.

70. Mandl $\mathrm{T}$, Meyerspeer $\mathrm{M}$, Reichel $\mathrm{M}$, et al. Functional electrical stimulation of long-term denervated, degenerated human skeletal muscle: Estimating activation using T2-parameter magnetic resonance imaging methods. Artif Organs 2008;32:604-8.

71. Meyerspeer M, Mandl T, Reichel M, et al. Effects of functional electrical stimulation in denervated thigh muscles of paraplegic patients mapped with T2 imaging. MAGMA. 2008;21:219-26.
72. Rossato E, Marziali A, Carraro U, et al. RISE2Italy Project: Muscle FES after peripheral nerve lesion. Basic Appl Myol 2009;19:169-72.

73. Zhi-Jun H, Jian H, Feng-Dong Z, et al. An assessment of the intra- and inter-reliability of the lumbar paraspinal muscle parameters using CT scan and magnetic resonance imaging. Spine 2011;36:E868-E874. doi: 10.1097/BRS. 0b013 e3181ef6b51.

74. Kalichman L, Hodges P, Li L, et al. Changes in paraspinal muscles and their association with low back pain and spinal degeneration: CT study. Eur Spine J 2010:19:1136-44.

75. Keller $\mathrm{A}$, Gunderson $\mathrm{R}$, Reikerås $\mathrm{O}$, et al. Reliability of Computed Tomography measurements of paraspinal muscle crosssectional area and density in patients with chronic low back pain. Spine 2003;28:1455-60.

76. Bret H. Goodpaster BH, Carlson CL, et al. Health $\mathrm{ABC}$ study. Attenuation of skeletal muscle and strength in the elderly: The Health ABC Study. J Appl Physiol 2001;90:2157-216.

77. Franzini Armstrong C, Gargiulo P, Stramare R. The EJTM Special "News on clinical imaging of muscle tissue" Eur J Transl Myol - Basic Appl Myol 2015;25:63-140.

78. Edmunds KJ, Gargiulo P. Imaging approaches in noninvasive functional assessment of implantable myogenic biomaterials and engineered muscle tissue. Eur J Transl Myol - Basic Appl Myol 2015;25:63-76.

79. Wiedemann L, Chaberova J, Edmunds KJ, et al. Low-amplitude craniofacial EMG power spectral density and 3D muscle reconstruction from MRI. Eur J Transl Myol - Basic Appl Myol 2015;25:93-9.

80. Magnússon B, Pétursson P, Kyle Edmunds KJ, et al. Improving planning and post-operative assessment for Total Hip Arthroplasty. Eur J Transl Myol - Basic Appl Myol 2015;25:101-8.

81. Ortolan P, Zanato R, Coran A, et al. Role of radiologic imaging in genetic and acquired neuromuscular disorders. Eur J Transl Myol Basic Appl Myol 2015; 25 (2): 121-7. 